\title{
Ton-Scale Production of
}

1,4-Bis(dichloromethyl)-2,5-dichlorobenzene via an Unexpected Controllable Chlorination of 1,4-Dichloro-2,5-dimethylbenzene

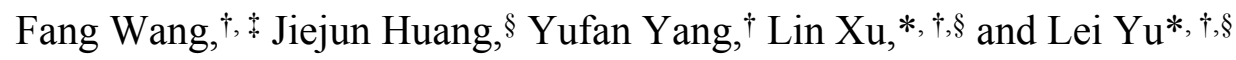

†School of Chemistry and Chemical Engineering, Yangzhou University, Yangzhou, Jiangsu 225002, P. R. China

\$Yangzhou Polytechnology Institute, Yangzhou, 225127, P. R. China

§Jiangsu Yangnong Chemical Group Co. Ltd., Yangzhou 225009, P. R. China Emails: 13773589268@139.com (L. Xu), yulei@yzu.edu.cn (L. Yu)

\section{CONTENTS}

${ }^{1} \mathrm{H}$ NMR of the product............................................. 2

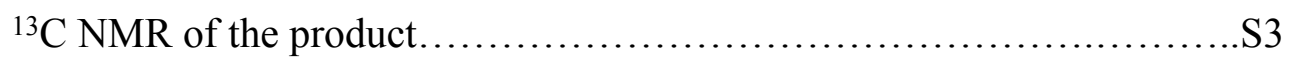

Quality analysis report.......................................... 4

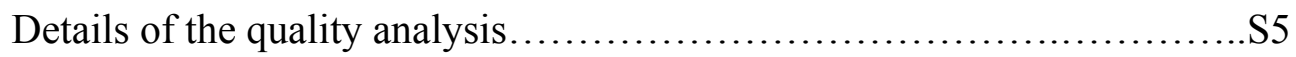


${ }^{1} \mathrm{H}$ NMR of the product<smiles>Clc1cc(C(Cl)Cl)c(Cl)cc1C(Cl)Cl</smiles>

1,4-Dichloro-2,5-bis-dichloromethyl-benzene (2), $\mathrm{CDCl}_{3}, 600 \mathrm{MHz}$

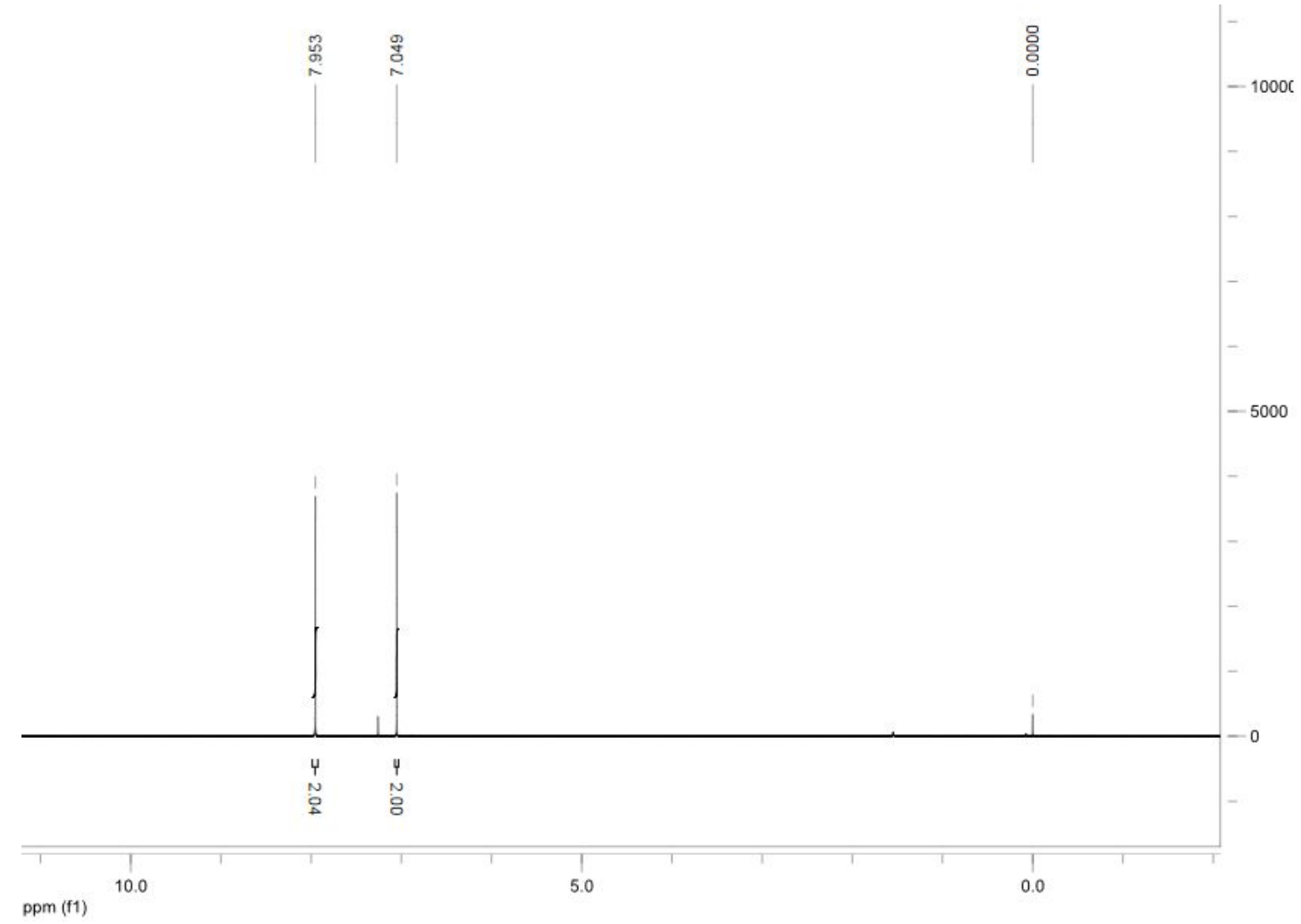


${ }^{13}$ C NMR of the product<smiles>Clc1cc(C(Cl)Cl)c(Cl)cc1C(Cl)Cl</smiles>

1,4-Dichloro-2,5-bis-dichloromethyl-benzene (2), $\mathrm{CDCl}_{3}, 150 \mathrm{MHz}$

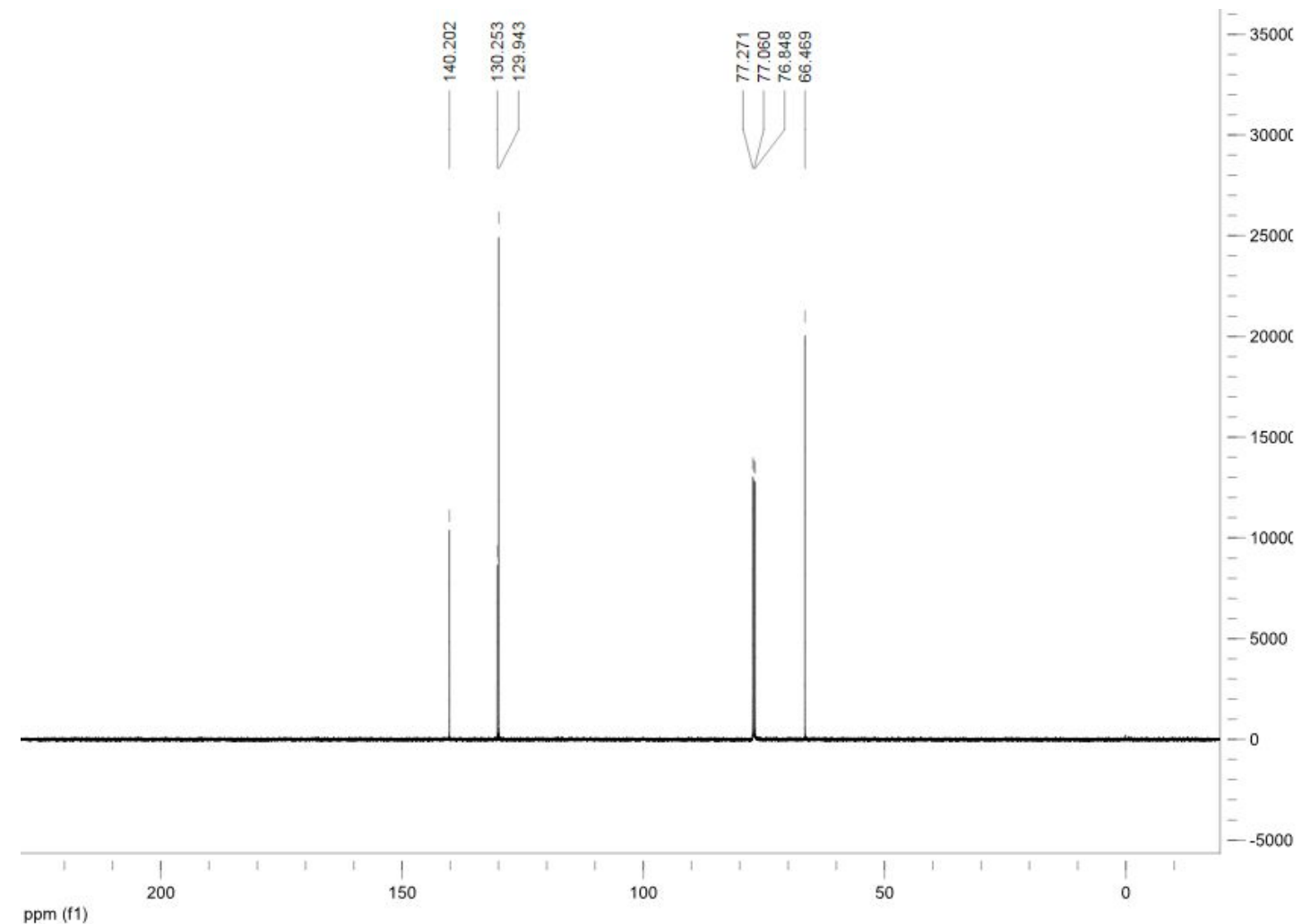




\section{Quality analysis report}

产品检验报告单

\section{Certificate Of Analysis}

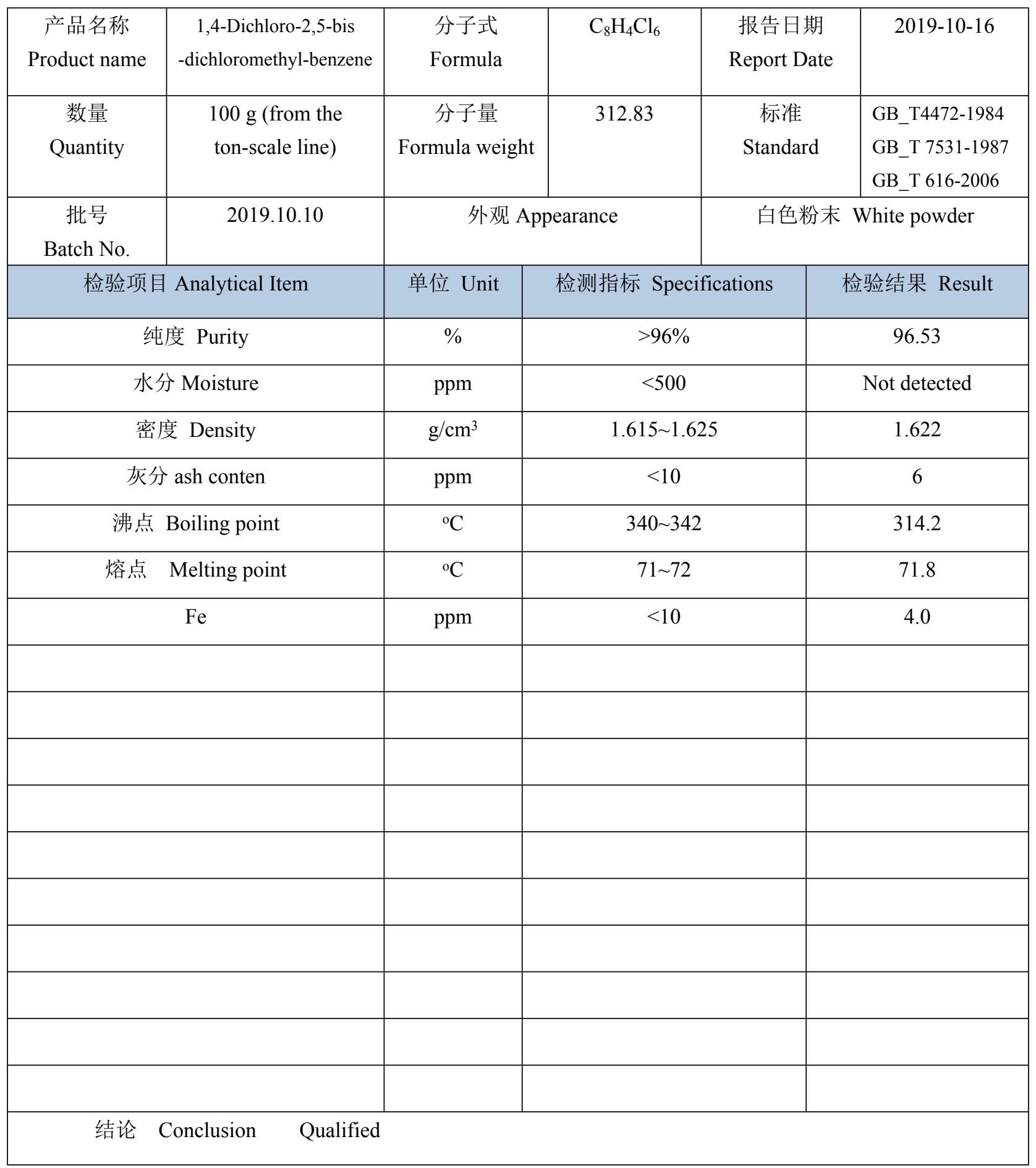




\section{Details of the quality analysis}

Tables S1 Methods of the product quality analysis ${ }^{a}$

\begin{tabular}{|c|c|c|c|}
\hline Entry & Item & Method & Remarks \\
\hline \multirow[t]{3}{*}{1} & Purity & Gas & \multirow{6}{*}{-} \\
\hline & & Chromatograph-External & \\
\hline & & Standard Normalization & \\
\hline 2 & Moisture & Karl Fischer method & \\
\hline \multirow[t]{2}{*}{3} & Density & Density-pyknometer & \\
\hline & \multicolumn{2}{|r|}{ method } & \\
\hline \multirow{2}{*}{4} & \multirow{2}{*}{ Ash content } & High-temperature & The ash content was \\
\hline & & incineration & weighted. \\
\hline 5 & Boiling point & Micro method & - \\
\hline 6 & Melting point & Capillary tube method & - \\
\hline \multirow[t]{3}{*}{7} & & Inductively coupled & \multirow{3}{*}{-} \\
\hline & $\mathrm{Fe}$ & plasma emission & \\
\hline & & spectrometry (ICPES) & \\
\hline
\end{tabular}

${ }^{a}$ Methods were performed according to GB_T4472-1984 (national standard), GB_T 7531-1987 and GB_T 616-2006(national standard) of China. 